\title{
Interplay between exchange interactions and charging effects in metallic grains.
}

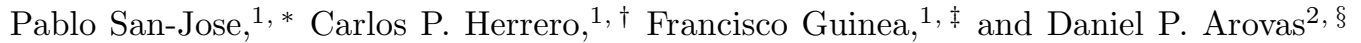 \\ ${ }^{1}$ Instituto de Ciencia de Materiales de Madrid. CSIC. Cantoblanco. 28049 Madrid. Spain \\ ${ }^{2}$ Department of Physics, University of California at San Diego, La Jolla California 92093, USA
}

\begin{abstract}
We study the effect of the exchange interactions in small superconducting grains near the Coulomb blockade regime. We extend the standard description of the grain in terms of a single collective variable, the charge and its conjugated phase, to include the spin degree of freedom. The suppression of spin fluctuations enhances the tendency towards Coulomb blockade. The effective charging energy and conductance are calculated numerically in the regime of large grain-lead coupling.
\end{abstract}

PACS numbers: 73.23.-b , 73.23.Hk

Introduction. Coulomb blockade in metallic grains is a well studied phenomenon [1, 2]. The transport through a grain in the Coulomb blockade regime can be studied using rate equations when the coupling to the leads is weak [1, 3]. The renormalization of the charging energy when the coupling to the leads is large is also well understood $[3,4,[5,6]$. The strongly coupled regime is best studied by introducing a single collective degree of freedom, the phase, conjugated to the total number of electrons in the grain $Q / e$ 7, $8,[9]$. The use of this variable is justified when the separation between the electronic levels within the grain can be neglected, or, alternatively, when the conductance of the grain is large. In this limit, the interaction effects within the grain can be described by a simple hamiltonian [10], expressed in terms of the total charge $\left(E_{C}\left(\hat{Q}-Q_{0}\right)^{2}\right)$, the total spin $\left(J_{S} \vec{S}^{2}\right)$ and the individual electronic degrees of freedom. In the presence of attractive interactions in the grain a pairing term $\left(\lambda_{B C S} \hat{T}^{+} \hat{T}\right)$ should also be included [11] that will drive the system towards superconducting state with energy gap $\Delta$. In such case both the pairing $\lambda_{B C S}$ and the exchange $J_{S}$ will grow under renormalization group (integration of energies down from Thouless energy) to a scale much larger than the bare one, which is initially of the order of the level spacing. Moreover due to the attractive character of interaction the spin susceptibility due to exchange will be positive, so that spin fluctuations get suppressed much in the same way as charge fluctuations do due to the charging energy. The essential distinction between the two will stem from the topological differences between spin group $\mathrm{SU}(2)$ and charge group $\mathrm{U}(1)$ in which their conjugate phases exist.

In the following, we will generalize the usual description of a small grain in the Coulomb blockade regime in terms of phase dynamics 7]. We include also the dynamics of the total spin of the grain in $\mathrm{SU}(2)$, on the same footing as the total charge. We assume that the grain has a negligible level spacing and a finite positive

\footnotetext{
*Electronic address: pablo.sanjose@icmm.csic.es

${ }^{\dagger}$ Electronic address: ch@icmm.csic.es

${ }^{\ddagger}$ Electronic address: paco.guinea@icmm.csic.es

$\S$ Electronic address: darovas@ucsed.edu
}

renormalized susceptibility according to the arguments above.

The coupling between the grain and an external (normal) electrode leads to single electron tunnelling. We describe it as usually done, in terms of a long range interaction, in time, between the phase and spin variables of the grain [12]. In most of the calculations, we neglect the long time cutoff in the kernel describing these interactions imposed by the superconducting gap. This approximation is reasonable when the bare charging energy is larger than the gap, and its renormalization arises from virtual tunnelling processes of high energy. The effects arising from the suppression of the subgap conductance due to the superconducting state can be incorporated into our calculations in a straightforward way. Finally, our method can be used to study superconducting junctions with subgap leakage currents [13], although we will not consider this case in detail.

The effects of a constant exchange term on the transport properties of a quantum dot has already been studied in the limit where the coupling to the leads is weak, using rate equations [14, 15, 16]. The present formalism goes one step beyond this by summing all processes up to cotunneling level.However we do not consider here the changes in the grain susceptibility induced by the spin-orbit coupling which has been considered by other authors [17.

The model. As mentioned above, we will focus on the case in which the superconducting gap is smaller or of similar order as the renormalized charging energy, $E_{C}^{*}$, and analyze how this renormalization of the bare charging energy, $E_{C}$, depends on the exchange J. These constraints are satisfied, for instance, by $\mathrm{Al}$ superconducting grains with radii below $100 \mathrm{~nm}$.

The hamiltonian that we study is: $\mathcal{H}=\mathcal{H}_{\text {grain }}+\mathcal{H}_{\text {lead }}+$ $\mathcal{H}_{\text {hop }}$, where

$$
\begin{aligned}
\mathcal{H}_{\text {grain }} & =\sum_{i, s} \epsilon_{i} d_{i, s}^{\dagger} d_{i, s}+E_{C} \hat{N}^{2}+J_{S} \vec{S}^{2} \\
\mathcal{H}_{\text {lead }} & =\sum_{k, s} \epsilon_{k} c_{k, s}^{\dagger} c_{k, s} \\
\mathcal{H}_{\text {hop }} & =-t \sum_{i, k, s} c_{i, s}^{\dagger} d_{k, s}+\text { h.c. }
\end{aligned}
$$


and $\hat{N}$ and $\vec{S}$ are the total number of electrons and the total spin of the grain, $\hat{N}=\sum_{i, s} d_{i, s}^{\dagger} d_{i, s}$ and $\vec{S}=$ $\frac{1}{2} \sum_{i, s, s^{\prime}} d_{i, s}^{\dagger} \vec{\sigma}_{s, s^{\prime}} d_{i, s^{\prime}}$, where $\vec{\sigma}$ denotes the Pauli matrices. The grain-dot conductance, in dimensionless units, can be approximated by $\alpha \approx t^{2} \rho_{\text {grain }}\left(\epsilon_{\mathrm{F}}\right) \rho_{\text {lead }}\left(\epsilon_{\mathrm{F}}\right)$, where $\rho_{\text {grain/lead }}\left(\epsilon_{\mathrm{F}}\right)$ is the density of states at the Fermi level of the grain and the lead. As mentioned above, we neglect the energy dependence of the density of states of the grain. The only interactions included in eq.(1) are through the total spin and charge of the grain.

Path integral formulation. We can integrate out the fermionic degrees of freedom and obtain a description in terms of collective variables only by using the path integral formalism. The action is $S=S_{\text {grain }}^{0}+S_{\text {lead }}+$ $S_{\text {hop }}+S_{\text {int }}$, where

$$
\begin{aligned}
S_{\text {grain }}^{0} & =\int_{0}^{\beta} d \tau \sum_{i, s} \bar{d}_{i, s}\left(\partial_{\tau}+\epsilon_{i}-\mu_{\text {grain }}\right) d_{i, s} \\
S_{\text {lead }} & =\int_{0}^{\beta} d \tau \sum_{k, s} \bar{c}_{k, s}\left(\partial_{\tau}+\epsilon_{k}-\mu_{\text {lead }}\right) c_{k, s} \\
S_{\text {hop }} & =-t \int_{0}^{\beta} d \tau \sum_{i, k, s} \bar{d}_{i, s} c_{k, s}+\text { H.c. } \\
S_{\text {int }} & =E_{C}\left(\hat{N}-N_{\text {ext }}\right)^{2}+J_{S}\left(\vec{S}-\vec{S}_{\text {ext }}\right)^{2}
\end{aligned}
$$

We have included an offset electron number $N_{\text {ext }}=$ $V_{\text {gate }} / e C_{g}$ induced by a gate voltage $V_{\text {gate }}$ and an offset spin $\vec{S}_{\text {ext }}=\vec{H}_{\text {ext }} / 2 J_{S}$ induced by an external magnetic field $\vec{H}_{\text {ext }}$, that couples to the total spin.

We can now decouple the quartic interaction term $S_{\text {int }}$ by means of a Hubbard-Stratonovich transformation $e^{-E c\left(N-N_{\text {ext }}\right)^{2}} \propto \int \mathcal{D} V e^{-V^{2} / 4 E_{\mathrm{C}}-i V\left(N-N_{\text {ext }}\right)}$, and similarly for $\vec{S}$, which introduces a new scalar field $V$ for the total charge and a vector field $\vec{H}$ for the total spin. We then have $\mathcal{S}_{\text {int }}=S_{0}+S_{1}$, with

$$
\begin{aligned}
& S_{0}=\int_{0}^{\beta} d \tau\left(\frac{V^{2}}{4 E_{C}}+\frac{\vec{H}^{2}}{4 J_{S}}-i V N_{\mathrm{ext}}-i \vec{H} \cdot \vec{S}_{\mathrm{ext}}\right) \\
& S_{1}=i \int_{0}^{\beta} d \tau(V \hat{N}+\vec{H} \cdot \vec{S}) .
\end{aligned}
$$

We now perform a time dependent canonical transformation (a phase and spin rotation) on the electronic wavefunctions, in order to cancel the term $S_{1}$ in eq.(3). This $U(1) \times S U(2)$ transformation can be written as:

$$
\begin{aligned}
d_{k s}(\tau) & \rightarrow U_{s s^{\prime}}(\tau) d_{k s^{\prime}}(\tau) \\
U(\tau) & =e^{i \phi(\tau)} e^{\frac{i}{2} \xi(\tau) \hat{n}(\tau) \cdot \vec{\sigma}}
\end{aligned}
$$

The transformation is parametrized by the angles $\phi(\tau)$ and $\xi(\tau)$, and by the three dimensional unitary vector $\hat{n}(\tau)$. The requirement that $S_{1}$ in (3) is cancelled implies:

$$
\left(\partial_{\tau} U\right) U^{\dagger}=i V+\frac{i}{2} \vec{H} \cdot \vec{\sigma}
$$

so that $V=\dot{\phi}$ and

$$
\vec{H}=\dot{\xi} \hat{n}+\sin \xi \dot{\hat{n}}+(1-\cos \xi) \dot{\hat{n}} \times \hat{n}
$$

These identities provide an alternative and convenient parametrization of the auxiliary fields $V$ and $\vec{H}$, represented now by the $\phi, \xi, \hat{n}$ fields, which will be used in the following. Note that the $U(1)$ gauge transformation needed to replace $V$ by the phase $\phi$ leads to the standard description of charging effects in terms of phase fluctuations. It is interesting to note that eq.(6) implies that $\vec{H}$ is proportional to the angular momentum of a sphere, considered as a rigid body [18]. The periodicity in imaginary time of the arguments in the action implies that $U(0)=U(\beta)$. This constraint implies the usual quantization of the charge in the grain, and also of the spin (see below), due to the discreteness of transport events.

A more compact notation for the transformation in eq.(4) can be given in terms of the following, $\tau$ dependent, two- and four-dimensional unit vectors:

$$
\begin{aligned}
& \hat{u}_{\tau}=\left(\sin \phi_{\tau}, \cos \phi_{\tau}\right) \\
& \hat{v}_{\tau}=\left(\hat{n}_{\tau} \sin \frac{1}{2} \xi_{\tau}, \cos \frac{1}{2} \xi_{\tau}\right)
\end{aligned}
$$

We can use these vectors to write $S_{0}$ as:

$$
S_{0}=\int_{0}^{\beta} d \tau\left\{\frac{\left(\partial_{\tau} \hat{u}\right)^{2}}{4 E_{C}}+\frac{\left(\partial_{\tau} \hat{v}\right)^{2}}{4 J_{S}}\right\}
$$

where external gates and fields have been taken as zero for the moment. The transformation in eq.(41) modifies also the lead-grain coupling, $S_{\mathrm{hop}}$ :

$$
S_{\text {hop }}=-t \int_{0}^{\beta} d \tau \sum_{i, k, s, s^{\prime}} \bar{c}_{i, s} U_{s, s^{\prime}} d_{k, s^{\prime}}+\text { H.c. }
$$

A final step to obtain the effective action for the rotor fields is to integrate out the fermionic fields to order $t^{2}$, using $\left\langle e^{-S_{\text {hop }}}\right\rangle_{0}=e^{-\frac{1}{2}\left\langle S_{\text {hop }}^{2}\right\rangle_{0}+\mathcal{O}\left(t^{4}\right)}$. One obtains the following dissipation term:

$$
S_{\text {diss }}=-\frac{\alpha}{4} \int_{0}^{\beta} d \tau \int_{0}^{\beta} d \tau^{\prime} K\left(\tau-\tau^{\prime}\right) \operatorname{Tr}\left[U_{\tau}^{\dagger} U_{\tau^{\prime}}+U_{\tau^{\prime}}^{\dagger} U_{\tau}\right]
$$

where $K(\tau)=-\left[G_{\text {lead }}(\tau) G_{\text {grain }}(-\tau)\right] /\left[\rho_{\text {lead }}\left(\epsilon_{\mathrm{F}}\right) \rho_{\text {grain }}\left(\epsilon_{\mathrm{F}}\right)\right]=$ $(\pi T)^{2} / \sin ^{2}(\pi T \tau), G_{\text {lead }}$ and $G_{\text {grain }}$ being the lead and grain unperturbed Green's functions in imaginary time. Recall here the that the finite range that the superconducting gap could bring in is assumed larger than the decay time of the phase correlators which is of order $E_{C}^{*}$, so that the gapless $K\left(\tau-\tau^{\prime}\right)$ of the normal state yields equivalent results. $S_{\text {diss }}$ may be finally recast as

$$
S_{\text {diss }}=\alpha \int_{0}^{\beta} d \tau \int_{0}^{\beta} d \tau^{\prime} K\left(\tau-\tau^{\prime}\right)\left[1-\left(\hat{u}_{\tau} \cdot \hat{u}_{\tau^{\prime}}\right)\left(\hat{v}_{\tau} \cdot \hat{v}_{\tau^{\prime}}\right)\right]
$$

This term is sufficient to account for second order tunnelling processes, and in particular it can describe cotunnelling features. The derivation is valid when the conductance between the grain and the electrode per channel is small, and it can be used even if the total value is large. 
The method leading to eq. (10) can be easily generalized to the case when the density of states in the grains or in the leads is spin dependent. The effective action will contain terms involving $\sin \left(\xi_{\tau}+\xi_{\tau^{\prime}}\right)$ which break the symmetry between the four components of the vector $\hat{v}_{\tau}$. These terms are analogous to the Josephson term which arises in the charge dynamics when the leads, or the grain are superconductors []].

The final action is $S_{\text {eff }}=S_{0}+S_{\text {diss }}$, written in terms of the dynamical variables $\hat{u}_{\tau}$ and $\hat{v}_{\tau}$ only. In the limit $J_{S}=0$, the field $\hat{v}_{\tau}$ can be taken as a constant, and the model reduces to the standard phase only model.

Results. It is instructive to analyze first the decoupled grain, described by $S_{0}$ in eq. (8), to see where this spherical rotor description of the total spin comes from. As mentioned above, $S_{0}$ contains the usual phase term, which leads to the quantization of the charge, and a contribution which is equivalent to that of a rigid rotor, and which leads to the conservation of spin. The eigenvalues associated to $S_{0}$ can be written as $E_{N, S, S_{z}, K}=$ $E_{C} N^{2}+J_{S} S(S+1)$, where $N=0,1,2 \cdots, S=0,1,2 \cdots$, $-S \leq S_{z} \leq S$ and $-S \leq K \leq S$. The degeneracy of a given state is $(2 S+1)^{2}$ [18]. This degeneracy can be understood by noting that, in the limit studied here, the level spacing within the grain can be neglected. The grain energy is solely determined by the total charge and the total spin. Let us assume that, in the neutral dot, there are $N_{0}$ spin $1 / 2$ electrons which contribute to the total spin. The number of states of total spin $S$ (each with degeneracy $2 S+1$ ) is:

$$
C_{S}^{N_{0}}=\left(\begin{array}{c}
N_{0} \\
\frac{N_{0}}{2}-S
\end{array}\right)-\left(\begin{array}{c}
N_{0} \\
\frac{N_{0}}{2}-S-1
\end{array}\right)
$$

In the limit of many electrons $N_{0} \rightarrow \infty$, one obtains $\lim _{N_{0} / S \rightarrow \infty} C_{S}^{N_{0}}=(2 S+1) C_{N_{0}}$, where $C_{N_{0}}=\frac{2^{N_{0}+3 / 2}}{\sqrt{\pi} N_{0}^{3 / 2}}$ is a constant independent of $S$. This means that the total degeneracy of a state composed of many $1 / 2$ spins and given value of the total spin momentum $\left\langle\hat{S}^{2}\right\rangle=S(S+1)$ is $C_{N_{0}}(2 S+1)^{2}$, just as $C_{N_{0}}$ rigid rotors with total angular momentum $S$. The existence of this degeneracy leads to a prefactor in the free energy which is independent of the angular momentum. This multiplicity, like similar degeneracies in the case of ordinary Coulomb blockade, does not affect the effects associated to the spin gap discussed in this paper.

The following calculations including the full action $S_{\text {eff }}$ have been done by averaging over all paths in the unit circle parameterized by $\hat{u}$ and the four-dimensional sphere which defines $\hat{v}$, using an extension of the Monte Carlo code developed earlier for related problems 6, 19]. The effective charging energy is calculated by summing over winding numbers of the phase. The conductance between the grain and the electrode has been approximated by the expression $G(\beta / 2)$ [19, 20], valid at low temperatures, where $G$ is the correlation function, in imaginary time, of the variable $\hat{v}_{\tau} \hat{u}_{\tau}$. The latter combination describes the transfer of a full electron to the grain. We

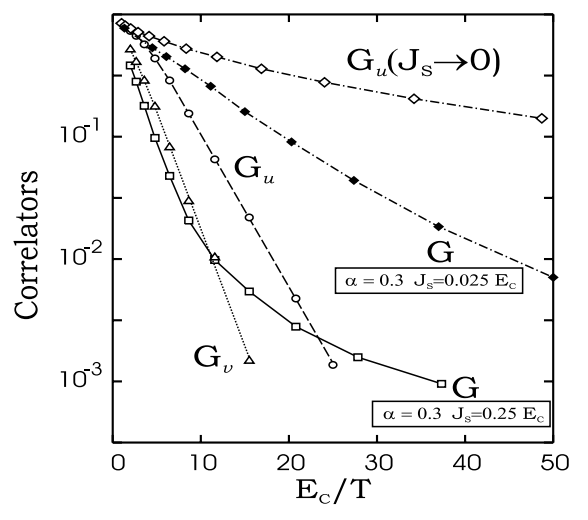

FIG. 1: Charge-charge, spin-spin and electron-electron current correlations (see text) versus inverse temperature for $\alpha=0.3$ and different values of $J_{S}$.

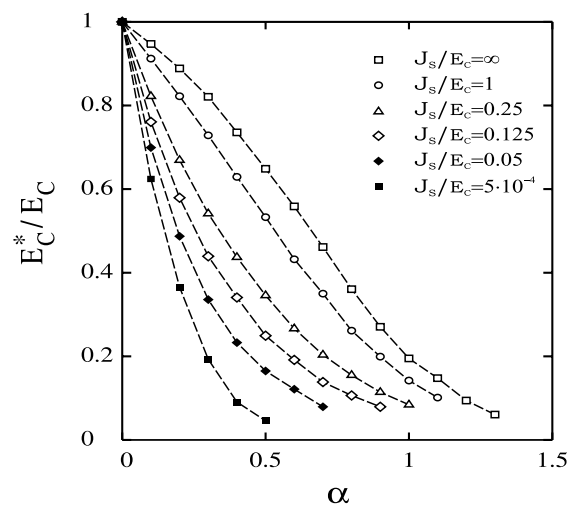

FIG. 2: Renormalized charging energy of the grain in the presence of a finite spin gap $J_{S}$, versus the dimensionless grainlead coupling $\alpha$. Note that the decay becomes less pronounced for growing spin gap.

calculate, separately, the correlations $G_{u}=\left\langle\hat{u}_{\tau} \cdot \hat{u}_{\tau^{\prime}}\right\rangle$ and $G_{v}=\left\langle\hat{v}_{\tau} \cdot \hat{v}_{\tau^{\prime}}\right\rangle$ which correspond to charge only and spin only currents.

The current correlation functions are shown in Fig. 11. It is interesting to note that both $\left\langle\hat{u}_{\tau} \cdot \hat{u}_{\tau^{\prime}}\right\rangle$ and $\left\langle\hat{v}_{\tau}\right.$. $\left.\hat{v}_{\tau^{\prime}}\right\rangle$ decay exponentially, while the composite correlation $\left\langle\hat{u}_{\tau} \cdot \hat{u}_{\tau^{\prime}} \hat{v}_{\tau} \cdot \hat{v}_{\tau^{\prime}}\right\rangle$ decays as $\left(\tau-\tau^{\prime}\right)^{-2}$, as required by Griffith's inequality [21]. The differences between the phasephase, "rotation-rotation" and current-current correlations is reminiscent of the behavior of a Luttinger liquid. It implies that the electron current cannot be factorized into its spin and charge components. The exponential decay of the correlations associated with the collective charge and spin degrees of freedom can be understood as the effect of a charge and spin gap in the grain. It can be obtained by making a mean field decoupling of the variables, in a similar way to the calculation for charging effects in coupled grains [22]. The $\left(\tau-\tau^{\prime}\right)^{-2}$ decay of the current-current correlation describes the cotunnelling processes at low temperatures.

The effective charging energies, as functions of $\alpha$ and $J_{S}$, are shown in Figs. 2] and [3. The effect of a finite $J_{S}$ 


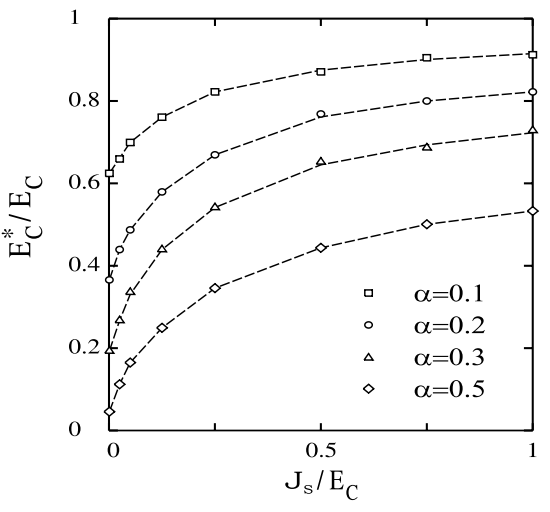

FIG. 3: Renormalized charging energy of the grain in the presence of a finite spin gap $J_{S}$, versus the value of the spin gap $J_{S}$. Note the saturation for large $J_{S}$.

on the renormalized charging energy is significant, even for small values of $J_{S}$. We can estimate analytically this effect, by assumming that when $J_{S} \rightarrow 0$ the fluctuations in the variable $\hat{v}_{\tau}$ are small. The effect of these fluctuations on the variable $\hat{u}_{\tau}$ can be approximated by replacing $\alpha$ in eq. (10) by $\alpha\left\langle|\hat{v}|^{2}\right\rangle$. Assuming that the fluctuations of $\hat{v}_{\tau}$ are harmonic, we find:

$$
\left\langle|\hat{v}|^{2}\right\rangle \approx 1-\int_{E_{C}}^{\Lambda} \frac{d \omega}{\omega^{2} / 2 J_{S}} \approx 1-\frac{J_{S}}{E_{C}}
$$

where $\Lambda$ is a high energy cutoff, comparable to the electronic bandwidth. Then, using the well known expression for the renormalized charging energy for large values of $\alpha$ [3, 4] : $E_{C}^{*} \approx E_{C} \exp \left\{-2 \pi^{2} \alpha\left(1-\frac{J_{S}}{E_{C}}\right)\right\}$. This enhancement of the effective charging energy by a spin gap is another manifestation of the non-separability of charge and spin.
Conclusions - We have analyzed the influence of the exchange term in a small superconducting grain on the charging effects in the regime where the superconducting gap is smaller or comparable to the charging energy. The suppression of the spin susceptibility reduces large fluctuations in the spin of the grain, and enhances the tendency towards Coulomb blockade. Our analysis integrates out the electronic degrees of freedom in the grain and in the external leads, and provides a simple description in terms of the charge and spin degrees of freedom of the grain only.

The effects of the exchange term have been analyzed for closed quantum dots, which are almost decoupled from the leads 14, 15, 16]. Our scheme provides a generalization which is non-perturbative in the coupling strength in the sense that one can recover exponential effects in the coupling, such as the renormalization of the charging energy, which cannot be derived from the addition of sequential processes.

A statistical approximation to the electron-electron interactions in a small dot predicts that the bare exchange term is negative and of the order of the separation between electronic levels [10, 23]. Our analysis, on the other hand, is valid only when the exchange term is positive and larger than the level spacing. This regime corresponds to systems with an attractive electron-electron interaction near a superconducting transition, when the exchange $J$ is significantly enhanced 24]. Spin fluctuations in a superconducting grain at low temperatures can therefore have a strong influence on charge fluctuations, restoring the system to a Coulomb blockade regime even when the coupling to the leads is strong.

Acknowledgements - Two of us (P. S. J. and F. G.) are thankful to MCyT (Spain) for financial support through grant MAT2002-0495-C02-01.
[1] D. V. Averin and K. K. Likharev, in Mesoscopic Phenomena in Solids, edited by B. L. Altshuler, P. A. Lee, and R. A. Webb (Elsevier, Amsterdam, 1991).

[2] H. Grabert and M. H. Devoret, eds., Single Electron Tunneling (Plenum, New York, 1992).

[3] G. Schön and A. D. Zaikin, Phys. Rep. 198, 238 (1990).

[4] F. Guinea and G. Schön, J. Low Temp. Phys. 69, 219 (1986).

[5] S. V. Panyukov and A. D. Zaikin, Phys. Rev. Lett. 67, 3168 (1991).

[6] C. P. Herrero, G. Schön, and A. D. Zaikin, Phys. Rev. B 59, 5728 (1999).

[7] V. Ambegaokar, U. Eckern, and G. Schön, Phys. Rev. Lett. 48, 1745 (1982).

[8] T.-L. Ho, Phys. Rev. Lett. 51, 2060 (1983).

[9] E. Ben-Jacob, E. Mottola, and G. Schön, Phys. Rev. Lett. 51, 2064 (1983).

[10] I. L. Kurland, I. L. Aleiner, and B. L. Altshuler, Phys. Rev. B 62, 14886 (2000).

[11] B. P. W. Aleiner, I. L. and L. I. Glazman, Phys. Rep.
358, 309 (2002)

[12] U. Eckern, G. Schön, and V. Ambegaokar, Phys. Rev. B 30, 6419 (1984).

[13] M. Iansiti, M. Tinkham, A. T. Johnson, W. F. Smith, and C. J. Lobb, Phys. Rev. B 39, 6465 (1989).

[14] Y. Alhassid, T. Rupp, A. Kaminski, and L. Glazman (2002), cond-mat/0212072.

[15] Y. Alhassid and T. Rupp, Phys. Rev. Lett. 91, 056801 (2003).

[16] G. Usaj and H. U. Baranger, Phys. Rev. B 67, 121308(R) (2003).

[17] K. A. Matveev, L. I. Glazman, and A. I. Larkin, Phys. Rev. Lett. 85, 2789 (2000).

[18] L. D. Landau and E. M. Lifschitz, Quantum Mechanics (Pergamon, Oxford, 1977).

[19] E. Bascones, C. P. Herrero, F. Guinea, and G. Schön, Phys. Rev. B 61, 16778 (2000).

[20] W. Zwerger and M. Scharpf, Zeit. Phys. B 85, 421 (1991).

[21] R. B. Griffiths, J. Math. Phys. 8, 478 (1967).

[22] D. P. Arovas, F. Guinea, C. P. Herrero, and P. SanJose, 
Phys. Rev. B 68, 085306 (2003).

[23] I. L. Aleiner, P. W. Brouwer, and L. I. Glazman, Phys. Rep. 358, 309 (2002).
[24] B. Mühlschlegel, D. J. Scalapino, and R. Denton, Phys. Rev. B 6, 1767 (1972). 